\title{
Análisis comparativo de las constantes vitales en pacientes agudos hospitalizados en función de la intensidad de dolor
}

\author{
L. Suso-Martî1,2, F. Cuenca-Martínez¹,2, A. Arredondo-López¹ y A. Gil-Martínez ${ }^{1-3}$
}

${ }^{1}$ Departamento de Fisioterapia. Centro Superior de Estudios Universitarios La Salle. Universidad Autónoma de Madrid. ${ }^{2} G r u p o$ de Investigación Motion in Brains. Instituto de Neurociencia y Ciencias del Movimiento (INCIMOV). Centro Superior de Estudios Universitarios La Salle. Universidad Autónoma de Madrid. ${ }^{3}$ Unidad de Fisioterapia. Hospital Universitario La Paz. Madrid. España

\section{ABSTRACT}

Objectives: A relevant aspect in hospitalized patient is pain intensity measurement. The objectives were firstly the identification of associated variables to pain and secondly if there was any association between the intensity of pain and the modification of the vital signs.

Materials and methods: The present research was a cross-sectional study was carried out in different areas of acute hospitalization in the University Hospital La Paz. Main variable was intensity of pain measured with numeric verbal scale, and secondary variables were vital signs, body temperature, systolic blood pressure, diastolic blood pressure, mean arterial pressure, heart rate, capillary blood glucose and oxygen saturation from the data obtained in the hospital monitoring instruments. The Student's T test was used for comparison between groups. The effect size was measured by the Cohen $\mathrm{d}$ and the association size by the Pearson $r$.

Results: A total of 180 patients were included. No statistically significant differences were found for any of the vital signs according to pain intensity levels ( $p>0.05$ ) divide in two groups. No statistically significant differences were found between vital signs as a function of pain intensity and the moment to collect measurements (morning/afternoon/evening) ( $p>0.05$ ). No statistically significant associations were found between pain intensity and vital signs except a statistically significant weak and negative association was observed between the diastolic blood

\section{RESUMEN}

Objetivos: El dolor es un aspecto de amplia repercusión en la calidad de vida de los pacientes y se ha propuesto incluirlo entre las constantes o signos vitales para que sea siempre recogido en la evaluación del paciente hospitalizado. El objetivo principal del presente estudio fue evaluar las constantes vitales en función de la intensidad de dolor en pacientes agudos hospitalizados, y el objetivo secundario fue observar si existen posibles asociaciones entre la intensidad del dolor y las constantes vitales.

Material y métodos: El presente estudio fue un estudio transversal realizado en diferentes áreas de hospitalización aguda en el Hospital Universitario La Paz. La variable principal fue la intensidad de dolor y las variables secundarias fueron las constantes vitales: temperatura corporal, tensión arterial sistólica, tensión arterial diastólica, tensión arterial media, frecuencia cardiaca, glucemia capilar y saturación de oxígeno. Se utilizó la prueba t de Student para comparación entre grupos. El tamaño del efecto se midió mediante la d de Cohen y el tamaño de asociación mediante la $r$ de Pearson.

Resultados: Se incluyeron un total de 180 pacientes. No se encontraron diferencias estadísticamente significativas para ninguna de las constantes vitales en función a los niveles de intensidad de dolor $(p>0,05)$ divididos en 2 grupos. Tampoco se hallaron diferencias estadísticamente significativas entre las constantes vitales en función de la intensidad de dolor en el turno de mañana, tarde y noche $(p>0,05)$. No se hallaron asociaciones estadísticamente significativas entre la intensidad de dolor y las constantes vitales, excepto 
pressure and the patients pain intensity $(r=-0.219$; $\mathrm{p}=0.032$ ].

Conclusions: Our results suggest that there are no differences between the vital signs according to the pain intensity and there are not associations between them.

Key words: Pain intensity, vital signs, acute pain, acute patient, in-patient. una asociación débil y negativa entre la tensión arterial diastólica y la intensidad de dolor percibido por los pacientes ( $r=-0,219 ; p=0,032$ ).

Conclusiones: En base a los resultados obtenidos en el presente estudio, no existen diferencias entre las constantes vitales en función la intensidad de dolor. Las variaciones de las constantes vitales parecen no ser una buena estimación de la intensidad de dolor en pacientes con dolor leve y dolor moderado-severo. Son necesarios análisis prospectivos en muestras de mayor tamaño para confirmar estos resultados.

Palabras clave: Intensidad de dolor, constantes vitales, paciente agudo, dolor agudo, paciente hospitalizado.

\section{INTRODUCCIÓN}

En la actualidad, el dolor es considerado como un gran problema de salud pública (1). La prevalencia del dolor crónico en España se estima según la literatura científica en un $17 \%$, presentando un gran impacto a nivel económico, social y personal (2). El dolor es la principal razón de consulta en los servicios de urgencias hospitalarios y, además, los pacientes hospitalizados presentan altas tasas del mismo $(3,4)$. La prevalencia de dolor en estos pacientes afecta de manera directa al proceso de hospitalización, retrasando la recuperación y, por tanto, incrementando la utilización de recursos hospitalarios (5).

La presencia de dolor en los pacientes hospitalizados está ampliamente relacionada con el proceso patológico del paciente, encontrando tasas elevadas en pacientes agudos, tanto por procesos de curación como en pacientes postquirúrgicos (6). Es por ello que la monitorización y control del dolor en los pacientes agudos hospitalizados se presenta como una medida relevante, siendo considerada la quinta constante vital (7). Los instrumentos más utilizados para evaluar la intensidad de dolor son las escalas subjetivas autoinformadas. Las escalas comúnmente utilizadas en la medición del dolor, tales como la visual analógica o la numérica verbal, ofrecen una valoración principalmente subjetiva del dolor y requieren de la colaboración del paciente y de su capacidad para comunicarse. Es por ello que, en la actualidad, se buscan otras medidas que permitan realizar una medición que ofrezca mayor objetividad y fiabilidad de la intensidad de dolor. Una de estas son las constantes vitales o variables fisiológicas, donde se ha sugerido que pueden servir como indicador de la actividad aumentada del sistema nervioso simpático-excitatorio ante la presencia de dolor (8). Debe considerarse que dicho sistema puede variar su actividad de acuerdo con la franja horaria en la que se evalúe y, por tanto, el dolor también podría hacerlo de la misma manera. Estas variables son utilizadas en los niños en edad preverbal debido a la imposibilidad de realizar medidas subjetivas autoinformadas y se han mostrado capaces de predecir el dolor agudo en esta población (9).

La hipótesis del presente estudio es que el dolor agudo podría modificar algunas de las constantes vitales y estas podrían ser utilizadas en la medición del dolor. Por tanto, el objetivo principal del presente estudio fue evaluar las constantes vitales en función de la intensidad de dolor en pacientes agudos hospitalizados, y el objetivo secundario fue analizar la relación entre la intensidad del dolor y las constantes vitales en pacientes con intensidad de dolor leve o moderadosevero.

\section{MATERIAL Y MÉTODOS}

\section{Diseño del estudio}

La presente investigación fue un estudio observacional trasversal siguiendo el diseño de la declaración STROBE para estudios observacionales (10). EI protocolo del estudio fue aprobado previamente por el Comité Ético de Investigación Clínica del Hospital Universitario La Paz (PI: 2283); una codificación que impidió la vulneración del derecho al anonimato de los participantes.

\section{Participantes}

Toda la información de los participantes se obtuvo en diferentes áreas de ingreso agudo del Hospital Universitario La Paz, de la Comunidad de Madrid. Los criterios de inclusión fueron los siguientes: a) pacientes agudos hospitalizados cuyo tiempo de estancia fuera igual o superior a 48 horas y, b) mayores de edad (> 18 años). La medición y el registro de todas las variables estudiadas fue realizada en el mismo momento. Los criterios de exclusión fueron los siguientes: a) pacientes ingresados en unidades crónicas, b) pacientes menores de edad y c) pacientes con diagnóstico médico de patología musculoesquelética crónica degenerativa. 


\section{Procedimientos}

La recogida de datos se realizó en el Hospital Universitario La Paz, de la Comunidad de Madrid, entre febrero y marzo de 2017. Se realizó un muestreo aleatorio simple entre los pacientes ingresados en unidades de agudos, en los años comprendidos desde el 2012 y el 2015 (tres últimos años disponibles en el momento del estudio], que cumpliesen los criterios de selección del estudio.

\section{Variable principal: intensidad del dolor}

La valoración de la intensidad de dolor se realizó a partir de los datos obtenidos por el personal sanitario mediante la escala verbal numérica (EVN) $(11,12)$, donde en una escala de 0 a 10, 0 es "sin dolor" y 10 es "el peor dolor imaginable", y donde se le pregunta al paciente cuál sería la puntuación que le pondría a su nivel de dolor en ese instante.

Se calculó la mediana de los resultados obtenidos para la variable intensidad de dolor $\left(m_{e}=4\right)$ con el objetivo de clasificar la muestra en función de los niveles de dolor percibido por los pacientes ingresados agudos, considerándose dolor leve aquel menor a 4 puntos en la EVN, y dolor moderado-severo aquel mayor o igual a 4 puntos. Se analizaron los datos de 180 pacientes, los cuales se repartieron en dos grupos, con dolor leve ( $n=87$ ) y con dolor moderado-severo ( $n=93$ ). Además, se dividió la muestra entre los turnos de mañana, tarde y noche con la finalidad de comprobar la existencia, o no, de diferencias en la intensidad de dolor en diferentes momentos de la franja horaria.

\section{Variables secundarias}

La temperatura corporal $\left(\mathrm{T}^{\mathrm{a}}\right)$ fue recogida en la región axilar y en grados centígrados (13) según los protocolos existentes.

La tensión arterial fue recogida y dividida en tres variables (la tensión arterial sistólica [TAS], la tensión arterial diastólica [TAD] y la tensión arterial media [TAM]), y se utilizó un fórmula estandarizada para calcularse: $[2 \times$ TAD + TAS]/3. Todas ellas utilizaron los milímetros de mercurio como unidad de medida (mmHg) (14).

La frecuencia cardiaca (FC) fue recogida en pulsaciones por minuto (15). La glucemia capilar (GC) en milígramos por decilitro (mg/dl) (16), y la saturación de oxígeno capilar (SatO2) fue recogida en porcentaje (17) según protocolos existentes.

La valoración de estas constantes vitales se realizó a través de los equipos de monitorización hospitalaria, monitor multiparamétrico Carescape V100 de Dinamap y Propaq CS de Welch Allyn, registrados según los protocolos del mismo y obteniendo la media de varias medidas.

\section{Cálculo del tamaño de la muestra}

Para el cálculo del tamaño muestral se utilizó un modelo de correlación normal bivariada correspon- diente al software de GPower de la Universidad de Düsseldorf (versión 3.1.9.2). Asumiendo un error $\alpha$ de 0,05, un poder estadístico del $95 \%$ y un posible tamaño de la asociación de $r=0,25$ (obtenido de un análisis piloto previo sobre 30 registros entre las variables intensidad de dolor y la TAD), serían necesarios 170 registros para detectar diferencias estadísticamente significativas.

\section{Análisis estadístico}

El paquete estadístico para ciencias sociales [SPSS 22, SPSS Inc., Chicago, IL, EE. UU.) fue el software que se utilizó para el análisis estadístico. El nivel de significación para todas las pruebas se estableció a $\mathrm{p}<0,05$. En el análisis de datos se ha utilizado estadística descriptiva para mostrar los datos de las variables continuas que se presentan como media \pm desviación típica (DT), intervalo de confianza (IC) del $95 \%$ y frecuencia relativa (porcentaje) en el caso de variables categóricas. Debido a que cada grupo estaba formado por más de 30 participantes, se decidió no realizar las pruebas de normalidad y utilizar test paramétricos de acuerdo con el teorema central del límite (18). Aun así, se comprobó que las variables seguían una distribución normal mediante una prueba de Kolmogorov-Smirnov. Se aplicó la prueba t de Student para muestras independientes como prueba estadística para comparar las variables continuas entre ambos grupos. Se calculó el tamaño del efecto (d de Cohen) para las variables estudiadas. De acuerdo con el método de Cohen, el efecto fue considerado como pequeño $[0,20$ a 0,49$)$, medio $[0,50$ a 0,79$)$ y grande $(>0,8)(19)$.

El coeficiente de correlación de Pearson se utilizó para comprobar las correlaciones entre variables cuantitativas. Un coeficiente de correlación de Pearson superior a 0,60 indica una fuerte correlación, un coeficiente entre 0,30 y 0,60 indica una moderada correlación, y un coeficiente menor a 0,30 una correlación débil.

\section{RESULTADOS}

En relación con los datos descriptivos, se encontraron diferencias estadísticamente significativas con respecto a la intensidad de dolor $(p<0,001)$, pero no se encontraron diferencias ni respecto a la edad, ni con respecto al género de los pacientes ( $p>0,05)$ (Tabla I).

No se encontraron diferencias estadísticamente significativas para ninguna de las constantes vitales en función a los niveles de intensidad de dolor $(p>0,05)$ (Tabla II). En adición a esto, se analizó si existían diferencias estadísticamente significativas entre las constantes vitales con respecto a la intensidad de dolor en función del turno en que se realizaron las mediciones. No se hallaron diferencias estadísticamente significativas entre las constantes vitales en función de la intensidad de dolor en el turno de mañana, tarde y noche ( $p>0,05$ ).

Finalmente, con respecto al análisis de correlaciones, no se encontraron correlaciones estadísticamente significativas entre las variables de edad, Ta, TAS, 
TABLA I

DATOS DESCRIPTIVOS DE LA MUESTRA

\begin{tabular}{|l|c|c|c|}
\hline \multicolumn{1}{|c|}{ Variables } & $\begin{array}{c}\text { Dolor leve } \\
(n=87)\end{array}$ & $\begin{array}{c}\text { Dolor moderado-severo } \\
(n=93)\end{array}$ & p-valor \\
\hline Edad (años) & $63,58 \pm 18,56$ & $67,18 \pm 17,29$ & 0,181 \\
\hline Dolor (EVN) & $3,00 \pm 0,76$ & $5,37 \pm 1,22$ & $<0,001^{\dagger}$ \\
\hline $\begin{array}{l}\text { Sexo } \\
\text { Hombre }\end{array}$ & $42(48,3)$ & $44(47,3)$ & 0,410 \\
Mujer & $45(51,7)$ & $49(52,7)$ & \\
\hline
\end{tabular}

${ }^{*} \mathrm{p}<0,05 .{ }^{\dagger} p<0,001$. Valores presentados como media \pm desviación típica y $n$ (\%). EVN: escala verbal numérica (0-10).

TABLA II

ANÁLISIS COMPARATIVO INTERSUBGRUPOS EN FUNCIÓN DE LA INTENSIDAD DE DOLOR MEDIANTE LA PRUEBA T DE STUDENT

\begin{tabular}{|l|c|c|c|c|c|c|c|}
\hline \multicolumn{1}{|c|}{ Variables } & $\begin{array}{c}\text { Dolor leve } \\
(n=87)\end{array}$ & $\begin{array}{c}\text { Dolor moderado- } \\
\text { severo } \\
(n=93)\end{array}$ & \multicolumn{3}{|c|}{$\begin{array}{c}\text { Diferencia de medias } \\
\text { (95\% \% IC) }\end{array}$} & p-valor & $\begin{array}{c}d \text { de } \\
\text { Cohen }\end{array}$ \\
\hline & & & Diferencia & Inferior & Superior & & \\
\hline $\mathrm{T}^{\mathrm{a}}\left({ }^{\circ} \mathrm{C}\right)$ & $36,11 \pm 0,59$ & $36,28 \pm 0,63$ & $-0,16$ & $-0,35$ & 0,01 & 0,08 & $\mathrm{~d}=-0,27$ \\
\hline TAS $(\mathrm{mmHg})$ & $122,38 \pm 20,48$ & $120,76 \pm 21,06$ & 1,61 & $-4,54$ & 7,77 & 0,60 & $\mathrm{~d}=0,08$ \\
\hline TAD $(\mathrm{mmHg})$ & $70,94 \pm 11,33$ & $67,83 \pm 11,61$ & 3,11 & $-0,28$ & 6,51 & 0,07 & $\mathrm{~d}=0,27$ \\
\hline TAM $(\mathrm{mmHg})$ & $88,08 \pm 13,27$ & $85,47 \pm 13,55$ & 2,60 & $-1,36$ & 6,58 & 0,19 & $\mathrm{~d}=0,19$ \\
\hline FC $(\mathrm{ppm})$ & $79,55 \pm 15,82$ & $79,24 \pm 15,27$ & 0,31 & $-4,28$ & 4,91 & 0,89 & $\mathrm{~d}=0,02$ \\
\hline $\mathrm{GC}(\mathrm{mg} / \mathrm{dl})$ & $123,27 \pm 34,45$ & $146,16 \pm 56,28$ & $-22,89$ & $-56,64$ & 10,86 & 0,06 & $\mathrm{~d}=-0,49$ \\
\hline $\mathrm{SO}_{2}(\%)$ & $94,94 \pm 3,28$ & $94,81 \pm 3,04$ & 0,13 & $-1,37$ & 1,64 & 0,85 & $\mathrm{~d}=0,04$ \\
\hline
\end{tabular}

${ }^{*} p<0,05 .{ }^{\dagger} p<0,001$. IC: intervalo de confianza. $T^{a}$ : temperatura. TAS: tensión arterial sistólica. TAD: tensión arterial diastólica. TAM: tensión arterial media. FC: frecuencia cardiaca. GC: glucemia capilar. SatO2: saturación de oxígeno capilar. milímetros de mercurio; ppm: pulsaciones por minuto.

TAM, FC, GC y SatO ${ }_{2}$ en relación con la intensidad de dolor percibido por los pacientes ingresados agudos ( $p>0,05)$. Sin embargo, se obtuvo una asociación estadísticamente significativa negativa débil entre la TAD y la intensidad de dolor percibido por los pacientes $(r=-0,219 ; p=0,032)$ (Tabla III).

\section{DISCUSIÓN}

El principal objetivo del presente estudio fue evaluar las constantes vitales en función de la intensidad de dolor en pacientes agudos hospitalizados. Los resultados de este estudio sugieren que no existen diferencias entre las constantes vitales en función de la intensidad del dolor en los pacientes agudos adultos hospitalizados, independientemente de que estos presenten dolor leve o dolor moderado-severo.

Los resultados obtenidos concuerdan con los descritos hasta el momento en gran parte de la literatura científica. Daoust y cols. realizaron un estudio de cohortes, donde no encontraron diferencias entre las constantes vitales y el dolor en pacientes del servicio de urgencias (20). Por otro lado, Bruijns y cols. realizaron una investigación en la que sometieron a sujetos
TABLA III

ANÁLISIS DE CORRELACIONES DE PEARSON

\begin{tabular}{|c|c|c|}
\hline & $\begin{array}{c}\text { Dolor leve } \\
(n=87)\end{array}$ & $\begin{array}{c}\text { Dolor } \\
\text { moderado- } \\
\text { severo } \\
(n=93)\end{array}$ \\
\hline & EVN (me $<4)$ & EVN (me $>4)$ \\
\hline Edad (años) & 0,091 & 0,035 \\
\hline$\left.T^{a} l^{\circ} \mathrm{C}\right)$ & 0,080 & $-0,006$ \\
\hline$T A S(m m H g)$ & $-0,058$ & $-0,074$ \\
\hline$T A D(m m H g)$ & $-0,219^{\star}$ & $-0,135$ \\
\hline$T A M(m m H g)$ & 0,125 & $-0,115$ \\
\hline$F C(p p m)$ & $-0,004$ & 0,039 \\
\hline$G C(m g / d l)$ & 0,188 & 0,117 \\
\hline$S a t O_{2}(\%)$ & $-0,104$ & $-0,191$ \\
\hline
\end{tabular}

${ }^{*} p<0,05 .{ }^{\dagger} p<0,001 . T^{a}:$ temperatura. TAS: tensión arterial sistólica. TAD: tensión arterial diastólica. TAM: tensión arterial media. FC: frecuencia cardiaca. GC: glucemia capilar. SatO ${ }_{2}$ : saturación de oxígeno capilar. ID (EVN): intensidad de dolor (escala verbal numérica). ppm: pulsaciones por minuto. me: mediana. 
sanos a un proceso doloroso agudo, encontrando que la percepción de dolor por parte de los participantes no se relacionó con una variación en las constantes vitales (21). Además, Ledowski y cols. no hallaron correlaciones significativas entre la intensidad de dolor postquirúrgico y los cambios hemodinámicos y neuroendocrinos registrados en las unidades de reanimación, sugiriendo que la ausencia de variación en las constantes vitales o en las variables autonómicas no debería interpretarse como garantía de la ausencia de dolor significativo [22].

Sin embargo, Bendall y cols. realizaron una investigación donde encontraron asociaciones entre las constantes vitales frecuencia respiratoria, FC y TAS con respecto a la intensidad de dolor, aunque estas fueron débiles. Además, obtuvieron que la frecuencia respiratoria aumentada era una variable predictora de la presencia de dolor severo (23). El valor de la media obtenida de la intensidad de dolor en el mencionado estudio fue de 8 puntos en la EVN, mientras que los valores de las medias obtenidas en el presente estudio fueron de 3 en el grupo de dolor leve, y 5,37 en el grupo de dolor moderado-severo, ambos menores a 8 puntos. En relación con esto, los resultados de una revisión de la literatura científica actual acerca de los procedimientos de evaluación de la intensidad de dolor en pacientes adultos en las unidades de cuidados intensivos sugiere que las variaciones de las constantes vitales en presencia de dolor severo representan de forma fiable de estimar la intensidad del mismo [24]. Por tanto, en base a los resultados del presente estudio y de los encontrados en evidencia científica actual, los autores sugieren que la utilización de las constantes vitales en presencia de dolor leve-moderado no son fiables para la predicción de la intensidad de este pero, sin embargo, existe controversia en presencia de dolor severo, debido a que se han encontrado estudios a favor, pero también en contra de esta estimación. Es, por lo tanto, que se requiere de mayor investigación y de mayor calidad metodológica para poder dar respuestas sólidas a esta cuestión.

Por otro lado, la medición de las constantes vitales en los pacientes infantiles recién nacidos también es un aspecto que ha sido investigado en la literatura científica, sugiriendo que sí son una forma fiable de estimar la intensidad de dolor, a diferencia de lo encontrado en el paciente adulto. De hecho, las constantes vitales, junto a los aspectos comportamentales en esta población de pacientes, son ampliamente utilizados en la evaluación de la intensidad de dolor infantil $(25,26)$. Los autores de este estudio sugieren que las diferencias entre la población infantil y la población adulta respecto a las diferencias entre las constantes vitales y la intensidad de dolor pueden estar motivadas por aspectos cognitivo-evaluadores y afectivo-emocionales presentes en mayor medida en el paciente adulto. Respecto a esto, Block y cols. encontraron que los pensamientos catastróficos presentan una mayor relación con respecto al dolor que las constantes vitales, dando por tanto una posible explicación a las diferencias encontradas en la validez de estas constantes entre el paciente adulto y el paciente infantil [27].

\section{LIMITACIONES DEL ESTUDIO}

En el presente estudio existen limitaciones que han de ser tenidas en cuenta en la interpretación de los resultados.

En primer lugar, la media de la intensidad de dolor en el grupo de dolor moderado-severo fue de 5,37 puntos en la EVN. Hubiera sido interesante obtener una muestra con mayores puntuaciones en la intensidad de dolor para así poder segmentarlo en dolor moderado (entre 3 y 6 puntos) y en dolor severo (> 6 puntos) debido a que, a pesar de que no existen, hasta la fecha, estudios que analicen las relaciones entre las constantes vitales y la intensidad de dolor en España, la principal controversia descrita en la literatura científica actual se encuentra en los pacientes agudos hospitalizados adultos que presentan dolor severo.

En segundo lugar, todos los pacientes incluidos en el presente estudio presentaban dolor agudo y estaban siendo sometidos a un tratamiento farmacológico analgésico según la intensidad del dolor presentada y la valoración médica pertinente; sin embargo, no se incluyeron registros de otros topos de medicación añadida por causas diferentes al tratamiento del dolor.

Por otro lado, deben tomarse con precaución los resultados de este trabajo, ya que tampoco se registraron la totalidad de las características clínicas de los pacientes ni tampoco el tratamiento exacto para su dolor, y que a su vez pudieran estar interfiriendo con la valoración de las constantes vitales.

Por último, debido al diseño del propio estudio, fue imposible poder determinar la hora exacta de los registros, así como la edad y/o experiencia de los profesionales que obtenían las variables no monitorizadas.

\section{CONCLUSIONES}

En base a los resultados obtenidos en el presente estudio, no existen diferencias entre las constantes vitales en función la intensidad de dolor. Las variaciones de las constantes vitales parecen no ser una estimación fiable de la intensidad de dolor en pacientes con dolor leve y dolor moderado-severo. Son necesarios análisis prospectivos para confirmar estos resultados.

\section{AGRADECIMIENTOS}

Los autores del estudio agradecen al Hospital Universitario La Paz la posibilidad de haber llevado a cabo el presente estudio.

\section{CONFLICTO DE INTERESES}

Los autores del presente estudio declaran no tener ningún conflicto de intereses.

\section{BIBLIOGRAFÍA}

1. Goldberg DS, McGee SJ. Pain as a global public health priority. BMC Public Health. 2011;11(1):770. DOI: 10.1186/14712458-11-770. 
2. Torralba A, Miquel A, Darba J. Situación actual del dolor crónico en España: iniciativa "Pain Proposal". Rev Soc Esp Dolor. 2014;21(1):16-22. doi: 10.4321/S113480462014000100003.

3. Todd KH, Ducharme J, Choiniere M, Crandall CS, Fosnocht DE, Homel P, et al. Pain in the Emergency Department: Results of the Pain and Emergency Medicine Initiative (PEMI) Multicenter Study. J Pain. 2007;8(6):460-6. DOI: 10.1016/j.jpain.2006.12.005.

4. Vallano A, Malouf J, Payrulet P, Baños JE, Catalan Research Group for Studying Pain in Hospital. Prevalence of pain in adults admitted to Catalonian hospitals: a cross-sectional study. Eur J Pain. 2006;10(8):721-31. DOI: 0.1016/j. ejpain.2005.11.003.

5. Levenson JL, Hamer RM, Rossiter LF. Psychopathology and pain in medical in-patients predict resource use during hospitalization but not rehospitalization. J Psychosom Res. 1992;36(6):585-92.

6. Rico P. El dolor y su impacto en la calidad de vida y estado anímico de pacientes hospitalizados. Univ Psychol Bogotá, Colomb. 2013;(1):55-62.

7. Timsit CA, Bouchene K, Olfatpour B, Herman P, Tran Ba Huy P. Epidemiology and clinical findings in 20,563 patients attending the Lariboisière Hospital ENT Adult Emergency Clinic. Ann Otolaryngol Chir Cervicofac. 2001;118(4):215-24.

8. Cowen R, Stasiowska MK, Laycock H, Bantel C. Assessing pain objectively: the use of physiological markers. Anaesthesia. 2015;70(7):828-47. DOI: 10.1111/anae.13018.

9. de Jesus JA, Tristao RM, Storm H, da Rocha AF, Campos D. Heart rate, oxygen saturation, and skin conductance: $A$ comparison study of acute pain in Brazilian newborns. 2011 Annu Int Conf IEEE Eng Med Biol Soc. 2011;1875-9. DOl: 10.1109/IEMBS.2011.6090532.

10. von Elm E, Altman DG, Egger M, Pocock SJ, Gotzsche PC, Vandenbroucke JP. Directrices para comunicación de estudios observacionales. Gac Sanit. 2008;22(2):144-50.

11. Hjermstad MJ, Fayers PM, Haugen DF, Caraceni A, Hanks $\mathrm{GW}$, Loge $\mathrm{JH}$, et al. Studies comparing numerical rating scales, verbal rating scales, and visual analogue scales for assessment of pain intensity in adults: A systematic literature review. J Pain Symptom Manage. 2011;41(6):1073-93. DOl: 10.1016/j.jpainsymman.2010.08.016.

12. Díez Burón F, Marcos Vidal JM, Baticón Escudero PM, Montes Armenteros A, Bermejo López JC, Merino García M. Concordancia entre la escala verbal numérica y la escala visual analógica en el seguimiento del dolor agudo postoperatorio. Rev Esp Anestesiol Reanim. 2011;58(5):279-82.

13. Romanovsky AA. Skin temperature: Its role in thermoregulation. Acta Physiol (Oxf). 2014;210(3):498-507.
14. MacDougall JD, Brittain M, MacDonald JR, McKelvie RS, Moroz DE, Tarnopolsky MA, et al. Validity of predicting mean arterial blood pressure during exercise. Med Sci Sports Exerc. 1999;31(12):1876-9.

15. Achten J, Jeukendrup AE. Heart rate monitoring: applications and limitations. Sports Med. 2003;33(7):517-38. DOl: 10.2165/00007256-200333070-00004.

16. Tablado MÁM, Martínez CM, Pérez JS. Nuevos dispositivos para determinar la glucemia. FMC Form Medica Contin en Aten Primaria. 2015;22(8):440-5.

17. Garde A, Karlen W, Dehkordi P, Ansermino JM, Dumont GA. Oxygen saturation resolution influences regularity measurements. In: 2014 36th Annual International Conference of the IEEE Engineering in Medicine and Biology Society. IEEE; 2014; 1:2257-60.

18. Tanushev MS, Arratia R. Central Limit Theorem for Renewal Theory for Several Patterns. J Comput Biol. 1997;4(1):3544. DOl: 10.1089/cmb.1997.4.35.

19. Cohen J. Statistical power analysis for the behavioral sciences. Lawrence Erlbaum Associates Inc., editor. Hillsdale; 1988.

20. Daoust R, Paquet J, Bailey B, Lavigne G, Piette É, Sanogo K, et al. Vital Signs Are Not Associated with Self-Reported Acute Pain Intensity in the Emergency Department. CJEM. 2016;18(01):19-27.

21. Bruijns SR, Guly HR, Wallis LA. Effect of spinal immobilization on heart rate, blood pressure and respiratory rate. Prehosp Disaster Med. 2013;28(3):210-4. DOI: 10.1017/ S1049023X13000034.

22. Ledowski T, Reimer M, Chavez V, Kapoor V, Wenk M. Effects of acute postoperative pain on catecholamine plasma levels, hemodynamic parameters, and cardiac autonomic control. Pain. 2012;153(4):759-64. DOl: 10.1016/j. pain.2011.11.002.

23. Bendall JC, Simpson PM, Middleton PM. Prehospital vital signs can predict pain severity. Eur J Emerg Med. 2011;18(6):3349. DOI: 10.1097/MEJ.Ob013e328344fdf2.

24. Gelinas C. Pain assessment in the critically ill adult: Recent evidence and new trends. Intensive and Critical Care Nursing. 2016;34:1-11. DOI: 10.1016/j.iccn.2016.03.001.

25. Hatfield LA, Ely EA. Measurement of acute pain in infants: a review of behavioral and physiological variables. Biol Res Nurs. 2015;17(1):100-11. DOI: $10.1177 / 1099800414531448$.

26. Witt N, Coynor S, Edwards C, Bradshaw H. A Guide to Pain Assessment and Management in the Neonate. Curr Emerg Hosp Med Rep. 2016;4(1):1-10. DOI: 10.1007/s40138016-0089-y.

27. Block PR, Thorn BE, Kapoor S, White J. Pain Catastrophizing, rather than Vital Signs, Associated with Pain Intensity in Patients Presenting to the Emergency Department for Pain. Pain Manag Nurs. 2017;18(2):102-9. D0l: 10.1016/j.pmn.2016.12.001. 